\title{
Sequences of 0's and 1's: sequence spaces with the separable Hahn property
}

\author{
by \\ MARia ZeLtser (Tallinn)
}

\begin{abstract}
In [3] it was discovered that one of the main results in [1] (Theorem 5.2), applied to three spaces, contains a nontrivial gap in the argument, but neither the gap was closed nor a counterexample was provided. In [4] the authors verified that all three above mentioned applications of the theorem are true and stated a problem concerning the topological structure of one of these three spaces. In this paper we answer the problem and give a counterexample to the theorem in doubt. Also we establish a new way of constructing separable Hahn spaces.
\end{abstract}

Let $\chi$ denote the set of all sequences of 0's and 1's and let $\chi(E)$ denote the linear hull of $\chi \cap E$. Given a sequence space $E$ we consider the natural order on it, i.e. for $x, y \in E$ with $x=\left(x_{k}\right), y=\left(y_{k}\right)$ we set $x \leq y$ whenever $x_{k} \leq y_{k}$ for every $k \in \mathbb{N}$. This order defines the positive cone

$$
E^{+}:=\{x \in E \mid x \geq 0\}=\left\{x \in E \mid x_{k} \geq 0(k \in \mathbb{N})\right\}
$$

on $E$.

For other notations and preliminary results we refer the reader to [1], [3] and [2].

1. Introduction. In [1] (see also [5] and [8]) the authors considered three types of Hahn properties. A sequence space $E$ is said to have the Hahn property, the separable Hahn property and the matrix Hahn property if the implication

$$
\chi(E) \subset F \Rightarrow E \subset F
$$

holds whenever $F$ is any FK-space, a separable FK-space and a matrix domain $c_{A}$ respectively. Evidently the Hahn property implies the separable Hahn property and the latter implies the matrix Hahn property. It was

2000 Mathematics Subject Classification: 46A45, 46A35, 40C05, 40H05.

Key words and phrases: Hahn property, separable Hahn property, matrix Hahn property, inclusion theorems, Hahn theorem, 0-1 sequences.

Supported by Project SF0132723s06. 
shown in [1, Theorem 5.3], and [8, Theorem 1.3] that the converse implications fail in general.

In [3] it was pointed out that the paper [1] by G. Bennett, J. Boos and $\mathrm{T}$. Leiger contains a nontrivial gap in the proof of Theorem 5.2. This theorem is one of the main results of the paper and it was applied three times (cf. [1, $(\mathrm{G})$ in Section 6, Theorem 6.4, Theorem 5.3]): for the space $|a c|_{0}$ of strongly almost-null sequences, $\ell^{\infty} \cap z^{\alpha}$ with $z \in \ell^{\infty} \backslash \ell^{1}$, and $\ell^{\infty}(|\lambda|)$, where $\left(\lambda_{k}\right)$ is an index sequence satisfying

$$
\lambda_{1}=1 \text { and } \sup _{k}\left(\lambda_{k+1}-\lambda_{k}\right)=\infty .
$$

In Theorem 5.2 of [1] the authors stated that for a monotone sequence space $E$ containing $\varphi$ the following conditions are equivalent:

(i) $E$ has the matrix Hahn property;

(ii) $E$ has the separable Hahn property;

(iii) $\chi(E)^{\beta}=E^{\beta}$.

However, in the proof of (iii) $\Rightarrow$ (ii) a false argument was used (see [3] for details).

In [3, Theorem 2] J. Boos and T. Leiger showed that the equivalence (i) $\Leftrightarrow$ (ii) holds for any monotone sequence space containing $\varphi$. Moreover, it is well known that (i) $\Rightarrow$ (iii) is valid for any sequence space $E$. So only the implication (iii) $\Rightarrow$ (ii) has not been settled.

In [7] it was shown that the theorem in doubt is true for $E=|a c|_{0}$ regardless of the validity of that theorem. In [4] a gliding hump argument was applied to show that two other applications (for $\ell^{\infty}(|\lambda|)$ and $\ell^{\infty} \cap z^{\alpha}$ ) of the theorem in [1] are valid.

We note that in the proof of the matrix Hahn property of $\ell^{\infty}(|\lambda|)$ the authors actually made use of the matrix Hahn property of $\ell^{\infty}$. Using the same idea of proof we will show in this paper that any sequence space

$$
X(|\lambda|, 1):=\left\{x \in \omega \mid\left(\sum_{k=\lambda_{n}}^{\lambda_{n+1}-1}\left|x_{k}\right|\right)_{n} \in X\right\}
$$

has the matrix Hahn property (even the separable Hahn property) whenever $X$ is positively solid and has the matrix Hahn property. This also gives us a way of constructing new separable Hahn spaces.

Now we will verify that the implication (iii) $\Rightarrow$ (ii) does not hold in general.

THEOREM 1.1. There exists a monotone sequence space E satisfying $\chi(E)^{\beta}=E^{\beta}$, but failing to have the matrix Hahn property.

Proof. Let $E:=\left\langle\left(c_{0 C_{1}} \cap \chi\right) \cup(\{x\} \cdot \chi)\right\rangle$, where $C_{1}$ is the Cesàro mean operator and $x$ is constructed as follows. Let $x_{k}=1 / k$ for $k=1, \ldots, \lambda_{1}$, 
where $\lambda_{1} \in \mathbb{N}$ is chosen such that

$$
\frac{1}{\lambda_{1}} \sum_{k=1}^{\lambda_{1}} x_{k} \leq 2^{-1}
$$

(this can be done since $C_{1}$ is regular). Now we set $x_{k}:=1-1 / k$ for $k=$ $\lambda_{1}+1, \ldots, \lambda_{2}$, where we choose $\lambda_{2}>\lambda_{1}$ such that

$$
\frac{1}{\lambda_{2}} \sum_{k=1}^{\lambda_{2}} x_{k} \geq 1-2^{-1}
$$

(here we also make use of the regularity of $C_{1}$ ). Proceeding in this way, for $i>1$ we set $x_{k}:=1 / k$ for $k=\lambda_{2 i-2}+1, \ldots, \lambda_{2 i-1}$, where $\lambda_{2 i-1}>\lambda_{2 i-2}$ is chosen such that

$$
\frac{1}{\lambda_{2 i-1}} \sum_{k=1}^{\lambda_{2 i-1}} x_{k} \leq 2^{-i}
$$

and then we set $x_{k}:=1-1 / k$ for $k=\lambda_{2 i-1}+1, \ldots, \lambda_{2 i}$, where $\lambda_{2 i}>\lambda_{2 i-1}$ is taken such that

$$
\frac{1}{\lambda_{2 i}} \sum_{k=1}^{\lambda_{2 i}} x_{k} \geq 1-2^{-i} .
$$

Evidently, $E$ is monotone, $E \subset \ell^{\infty}$ and it can be verified that $E \cap \chi=$ $c_{0 C_{1}} \cap \chi$.

We will prove that $E^{\beta}=(E \cap \chi)^{\beta}$. First we note that since $\mathcal{T} \subset E \cap$ $\chi \subset \ell^{\infty}$ and $\mathcal{T}^{\beta}=\ell^{1}$, we have $(E \cap \chi)^{\beta}=\ell^{1}$. In view of the inclusions $E \cap \chi \subset E \subset \ell^{\infty}$, also $E^{\beta}=\ell^{1}$. On the other hand, in view of (1.2) and (1.3), $x \notin c_{C_{1}}$, so $E \not \subset c_{C_{1}}$.

Hence the implication (iii) $\Rightarrow$ (ii) of Theorem 5.2 in [1] does not hold.

Now coming back to the spaces $\ell^{\infty} \cap z^{\alpha}$ and $\ell^{\infty}(|\lambda|)$, we answer the problem stated in [4]. It was shown there that both $\ell^{\infty} \cap z^{\alpha}$ and $\ell^{\infty}(|\lambda|)$ as well as their $\beta$-dual spaces are solid BK-spaces. Moreover, the linear functional defined by any element $y$ of the $\beta$-dual was shown to be continuous. For $\ell^{\infty}(|\lambda|)$ the authors proved that the norm of this functional is equal to the norm of $y$ in the $\beta$-dual space while for $\ell^{\infty} \cap z^{\alpha}$ they only succeeded in verifying that the norm of the functional is less than or equal to the norm of $y$. So they asked whether equality holds. The following example demonstrates that the answer is negative.

ExAMPLE. We use the notation of [4]:

$$
E:=\ell \cap z^{\alpha} \quad \text { and } \quad F:=E^{\beta}=\ell^{1}+\ell^{\infty} \cdot\{z\} .
$$

We consider

$$
z=\left(\frac{1}{k}\right) \quad \text { and } \quad y=\left(\frac{1}{2^{k}}+\frac{1}{k}\right) .
$$


Evidently $y \in \ell^{1}+\ell^{\infty} \cdot\{z\}$. To evaluate $\|y\|_{F}$, consider a representation $y=v+w z$, where

$$
v=\left(\frac{1}{2^{k}}-\frac{\alpha_{k}}{k}\right) \in \ell^{1} \quad \text { and } \quad w=\left(1+\alpha_{k}\right) \in \ell^{\infty} .
$$

For $\alpha_{k}=k / 2^{k}(k \in \mathbb{N})$ we get $\|w\|_{\infty}+\|v\|_{1}=3 / 2$, so $\|y\|_{F} \leq 3 / 2$. We will show that $\|w\|_{\infty}+\|v\|_{1} \geq 3 / 2$ for $\left(\alpha_{k}\right) \neq\left(k / 2^{k}\right)$, hence $\|y\|_{F}=3 / 2$ follows. Note that for all $\left(\alpha_{k}\right)$ we get

$$
\|w\|_{\infty}+\|v\|_{1}=\sup _{k}\left|1+\alpha_{k}\right|+\sum_{k}\left|\frac{1}{2^{k}}-\frac{\alpha_{k}}{k}\right| \geq\left|1+\alpha_{1}\right|+\left|\frac{1}{2}-\alpha_{1}\right| .
$$

If $\alpha_{1} \in[-1,1 / 2]$, then $\left|1+\alpha_{1}\right|+\left|1 / 2-\alpha_{1}\right|=1+\alpha_{1}+1 / 2-\alpha_{1}=3 / 2$. For $\alpha_{1}<-1$ we get $\left|1+\alpha_{1}\right|+\left|1 / 2-\alpha_{1}\right|=-2 \alpha_{1}-1 / 2>3 / 2$ and for $\alpha_{1}>1 / 2$ we get $\left|1+\alpha_{1}\right|+\left|1 / 2-\alpha_{1}\right|=2 \alpha_{1}+1 / 2>3 / 2$. So $\|y\|_{F}=3 / 2$.

In order to determine the operator norm $\left\|f_{y}\right\|$ of $f_{y}$ we fix $x \in E$ with $\|x\|_{E}=\|x\|_{\infty}+\sum_{k}\left|x_{k} / k\right|=1$. We estimate

$$
\begin{aligned}
\left|\sum_{k} x_{k} y_{k}\right| & \leq \sum_{k} \frac{\left|x_{k}\right|}{k}+\sum_{k} \frac{\left|x_{k}\right|}{2^{k}} \leq \sum_{k} \frac{\left|x_{k}\right|}{k}+\|x\|_{\infty} \sum_{k} \frac{1}{2^{k}} \\
& =\sum_{k} \frac{\left|x_{k}\right|}{k}+\|x\|_{\infty}=1 .
\end{aligned}
$$

Therefore $\left\|f_{y}\right\| \leq 1<\|y\|_{F}$.

2. Generalization of $\ell^{\infty}(|\lambda|)$. Throughout this section we assume that $1 \leq p, q \leq \infty$. For $p \in(1, \infty)$ we define $p^{\prime}$ to be the number satisfying $1 / p+1 / p^{\prime}=1$. We also use the usual convention that $p^{\prime}=\infty$ for $p=1$ and $p^{\prime}=1$ for $p=\infty$. Most of the proofs in this section are carried out for $p \in(1, \infty)$. The argument for the cases $p=1$ and $p=\infty$ is analogous.

Given a subset $X$ of $\omega$, an index sequence $\left(\lambda_{k}\right)$ satisfying (1.1) and $p$ with $1 \leq p \leq \infty$ we define

$$
X(|\lambda|, p):=\left\{x \in \omega \mid T^{p}(x):=\left(\left(\left\|x^{\left[\lambda_{n+1}-1\right]}-x^{\left[\lambda_{n}-1\right]}\right\|_{p}\right)_{n}\right) \in X\right\} .
$$

Note that setting $X=\ell^{q}$ we obtain the space $\ell(\lambda, p, q)$ introduced in [6].

If $X$ is a sequence space, then $X(|\lambda|, p)$ is closed under scalar multiplication. To guarantee that $X(|\lambda|, p)$ is also closed under vector addition, we need to demand that $X$ is positively solid, i.e., satisfies the condition

$$
u \in X, 0 \leq v \leq u \Rightarrow v \in X .
$$

Indeed, if $X$ is positively solid and $x, y \in X(|\lambda|, p)$, then by the Minkowski inequality,

$$
0 \leq T^{p}(x+y) \leq T^{p}(x)+T^{p}(y)
$$


hence $T^{p}(x+y) \in X$, implying $x+y \in X(|\lambda|, p)$. So if $X$ is a positively solid sequence space, then $X(|\lambda|, p)$ is a sequence space.

On the other hand, we will show that if $X$ is not positively solid, then $X(|\lambda|, p)$ is not a vector space . Indeed, by assumption we can find $u, v \in \omega$ with $0 \leq v \leq u$ and $u \in X$, but $v \notin X$. We set $x_{\lambda_{n}}:=v_{n}, x_{\lambda_{n}+1}:=$ $\left(u_{n}^{p}-v_{n}^{p}\right)^{1 / p}, y_{\lambda_{n}}:=v_{n}, y_{\lambda_{n}+1}:=-\left(u_{n}^{p}-v_{n}^{p}\right)^{1 / p}(n \in \mathbb{N})$ and $x_{k}:=y_{k}:=0$ for $k \notin\left\{\lambda_{n}, \lambda_{n}+1 \mid n \in \mathbb{N}\right\}$. Then

$$
\left(\sum_{k=\lambda_{n}}^{\lambda_{n+1}-1}\left|x_{k}\right|^{p}\right)^{1 / p}=\left(\sum_{k=\lambda_{n}}^{\lambda_{n+1}-1}\left|y_{k}\right|^{p}\right)^{1 / p}=u_{n} \quad(n \in \mathbb{N}) .
$$

So $x$ and $y$ are in $X(|\lambda|, p)$. On the other hand,

$$
\left(\sum_{k=\lambda_{n}}^{\lambda_{n+1}-1}\left|x_{k}+y_{k}\right|^{p}\right)^{1 / p}=2 v_{n} \quad(n \in \mathbb{N})
$$

so $x+y \notin X(|\lambda|, p)$. Hence $X(|\lambda|, p)$ is not a vector space.

Evidently any solid space is positively solid. On the other hand, $b s$ and $c s$ are positively solid spaces which are not solid. It is easy to verify that a sequence space is solid if and only if it is monotone and positively solid. Note also that if $X$ is positively solid, then $X(|\lambda|, p)$ is solid. Hereafter we suppose that $X$ is positively solid and contains $\varphi$. Hence the space $\left\langle X^{+}\right\rangle$is solid.

Evidently, $X(|\lambda|, p)=X^{+}(|\lambda|, p)$. Hence in particular $\ell(|\lambda|, p)=c s(|\lambda|, p)$ $=b s(|\lambda|, p)$ and more generally, $X_{1}(|\lambda|, p)=X_{2}(|\lambda|, p)$ if $X_{1}^{+}=X_{2}^{+}$.

Proposition 2.1. Let $\xi \in\{\alpha, \beta, \gamma\}$. Then $(X(|\lambda|, p))^{\xi}=\left(X^{+}\right)^{\alpha}\left(|\lambda|, p^{\prime}\right)$.

Proof. Since $X(|\lambda|, p)$ is solid, it is sufficient to show that $(X(|\lambda|, p))^{\alpha}=$ $\left(X^{+}\right)^{\alpha}\left(|\lambda|, p^{\prime}\right)$. Let $y \in\left(X^{+}\right)^{\alpha}\left(|\lambda|, p^{\prime}\right)$ and $x \in X(|\lambda|, p)$. Then by Hölder's inequality,

$$
\begin{aligned}
\sum_{k}\left|y_{k} x_{k}\right| & \leq \sum_{n}\left(\sum_{k=\lambda_{n}}^{\lambda_{n+1}-1}\left|x_{k}\right|^{p}\right)^{1 / p}\left(\left.\sum_{k=\lambda_{n}}^{\lambda_{n+1}-1}\left|y_{k}\right|\right|^{\prime}\right)^{1 / p^{\prime}} \\
& =\sum_{n}\left[T^{p}(x)\right]_{n}\left[T^{p^{\prime}}(y)\right]_{n}<\infty .
\end{aligned}
$$

Hence $(X(|\lambda|, p))^{\alpha} \supset\left(X^{+}\right)^{\alpha}\left(|\lambda|, p^{\prime}\right)$.

Now suppose, contrary to our claim, that there exists $y \in(X(|\lambda|, p))^{\alpha} \backslash$ $\left(X^{+}\right)^{\alpha}\left(|\lambda|, p^{\prime}\right)$. Then $u:=T^{p^{\prime}}(y) \notin\left(X^{+}\right)^{\alpha}$, so we can find $z \in X^{+}$such that $\sum_{n}\left|u_{n} z_{n}\right|=\sum_{n} u_{n} z_{n}=\infty$. We choose a sequence $\left(\varepsilon_{n}\right)$ with $0<\varepsilon_{n}<u_{n}$ $(n \in \mathbb{N})$ such that $\sum_{n} \varepsilon_{n} z_{n}<1$. 
For every $n \in \mathbb{N}$ we consider the functional

$$
f_{n}: l_{p}^{\lambda_{n+1}-\lambda_{n}} \rightarrow \mathbb{R}, \quad f_{n}(t)=\sum_{k=\lambda_{n}}^{\lambda_{n+1}-1} y_{k} t_{k-\lambda_{n}+1} .
$$

These functionals are continuous and satisfy

$$
\left\|f_{n}\right\|=\left\|\left(y_{k}\right)_{k=\lambda_{n}}^{\lambda_{n+1}-1}\right\|_{p^{\prime}}=\left[T^{p^{\prime}}(y)\right]_{n}=u_{n} \quad(n \in \mathbb{N}) .
$$

So we can find $x \in \omega$ such that

$$
\left[T^{p}(x)\right]_{n}=z_{n} \quad \text { and } \quad\left|f_{n}\left(\left(x_{k}\right)_{k=\lambda_{n}}^{\lambda_{n+1}-1}\right)\right|>\left(\left[T^{p^{\prime}}(y)\right]_{n}-\varepsilon_{n}\right) z_{n} \quad(n \in \mathbb{N}) .
$$

Hence $T^{p}(x)=z \in X^{+} \subset X$, therefore $x \in X(|\lambda|, p)$. On the other hand, $\sum_{k}\left|x_{k} y_{k}\right| \geq \sum_{n}\left|\sum_{k=\lambda_{n}}^{\lambda_{n+1}-1} x_{k} y_{k}\right| \geq \sum_{n}\left(\left[T^{p^{\prime}}(y)\right]_{n}-\varepsilon_{n}\right) z_{n} \geq \sum_{n} u_{n} z_{n}-1=\infty$, hence $y \notin(X(|\lambda|, p))^{\alpha}$. This contradiction proves that $y \in\left(X^{+}\right)^{\alpha}\left(|\lambda|, p^{\prime}\right)$. Hence $(X(|\lambda|, p))^{\alpha}=\left(X^{+}\right)^{\alpha}\left(|\lambda|, p^{\prime}\right)$.

Lemma 2.2. Let $\left(X, \tau_{X}\right)$ be a $K$-space with the topology generated by the system of seminorms $\{p \mid p \in \mathcal{P}\}$. Then $X(|\lambda|, q)$ is a K-space with the topology $\tau$ generated by the system of seminorms $\{\tilde{p} \mid p \in \mathcal{P}\}$ defined by

$$
\tilde{p}(x)=p\left(T^{q}(x)\right) \quad(x \in X(|\lambda|, q) ; p \in \mathcal{P}) .
$$

Proof. To show that $X(|\lambda|, q)$ is a K-space, we suppose that $\left(x^{(n)}\right)$ converges to $x$ in $(X(|\lambda|, q), \tau)$. Then, since $X$ is a $\mathrm{K}$-space, we have

$$
\left[T^{q}\left(x^{(n)}-x\right)\right]_{i}=\left(\sum_{k=\lambda_{i}}^{\lambda_{i+1}-1}\left|x_{k}^{(n)}-x_{k}\right|^{q}\right)^{1 / q} \rightarrow 0 \quad \text { as } n \rightarrow \infty(i \in \mathbb{N}) .
$$

Since

$$
\left|x_{k}^{(n)}-x_{k}\right| \leq\left(\sum_{j=\lambda_{i}}^{\lambda_{i+1}-1}\left|x_{j}^{(n)}-x_{j}\right|^{q}\right)^{1 / q} \quad\left(\lambda_{i} \leq k<\lambda_{i+1} ; i, k \in \mathbb{N}\right),
$$

the K-property of $(X(|\lambda|, q), \tau)$ follows.

In order to spread an FK-property from $X$ to $X(|\lambda|, q)$, we assume that the topology of $X$ is consistent with the natural order. More precisely, we asssume that seminorms $\left\{p_{k}\right\}$ generating the FK-topology of $X$ satisfy

$$
u, v \in X, 0 \leq u \leq v \Rightarrow p_{k}(u) \leq p_{k}(v) \quad(k \in \mathbb{N}) .
$$

Moreover, we require the condition

$$
p_{k}(u)=\sup _{n} p_{k}\left(u^{[n]}\right) \quad\left(k \in \mathbb{N} ; u \in X^{+}\right) .
$$

Note that (2.5) is stronger than the AB-property and, on the assumption that (2.4) is satisfied, weaker than the AK-property. Obviously the norms 
\|\|$_{q}(1 \leq q \leq \infty)$ and \|\|$_{b s}$ satisfy both (2.4) and (2.5) while \|\|$_{b v}$ fails to have both (2.4) and (2.5).

Proposition 2.3. Let $X$ be an FK-space with the topology $\tau_{X}$ generated by a system of seminorms $\left\{p_{k}\right\}$ satisfying (2.4) and (2.5). Then $X(|\lambda|, q)$ is an FK-space with the topology $\tau$ generated by the system of seminorms $\left\{\tilde{p}_{k}\right\}$ defined by

$$
\tilde{p}_{k}(x)=p_{k}\left(T^{q}(x)\right) \quad(x \in X(|\lambda|, q) ; k \in \mathbb{N}) .
$$

Proof. In view of Lemma 2.2 it is sufficient to prove that $(X(|\lambda|, q), \tau)$ is complete. Suppose that $\left(x^{(n)}\right)$ is a Cauchy sequence in $(X(|\lambda|, q), \tau)$. By the K-property of $(X(|\lambda|, q), \tau)$ the sequence $\left(x_{k}^{(n)}\right)$ is a Cauchy sequence for every $k \in \mathbb{N}$, hence $\left(x^{(n)}\right)$ converges coordinatewise to some $x \in \omega$. Since

$$
\left|\left[T^{q}\left(x^{(m)}\right)\right]_{i}-\left[T^{q}\left(x^{(n)}\right)\right]_{i}\right| \leq\left[T^{q}\left(x^{(m)}-x^{(n)}\right)\right]_{i} \quad(i \in \mathbb{N}),
$$

(2.4) implies that $T^{q}\left(x^{(n)}\right)$ is a Cauchy sequence in $\left(X, \tau_{X}\right)$, hence converges to some $z \in X$. By the K-property of $\left(X, \tau_{X}\right)$ we have

$$
\left[T^{q}\left(x^{(n)}\right)\right]_{i}=\left(\sum_{k=\lambda_{i}}^{\lambda_{i+1}-1}\left|x_{k}^{(n)}\right|^{q}\right)^{1 / q} \rightarrow z_{i} \quad \text { as } n \rightarrow \infty(i \in \mathbb{N}) .
$$

On the other hand, by the K-property of $(X(|\lambda|, q), \tau)$ it follows that

$$
\left(\sum_{k=\lambda_{i}}^{\lambda_{i+1}-1}\left|x_{k}^{(n)}\right|^{q}\right)^{1 / q} \rightarrow\left(\sum_{k=\lambda_{i}}^{\lambda_{i+1}-1}\left|x_{k}\right|^{q}\right)^{1 / q} \quad \text { as } n \rightarrow \infty(i \in \mathbb{N}) .
$$

Hence $T^{q}(x)=z \in X$, implying $x \in X(|\lambda|, q)$. Now we prove that $x^{(n)} \rightarrow x$ in $(X(|\lambda|, q), \tau)$. We set $u^{(n)}:=T^{q}\left(x^{(n)}-x\right)(n \in \mathbb{N})$. Let $\varepsilon>0$ and $k, s \in \mathbb{N}$. We choose $N \in \mathbb{N}$ such that $p_{k}\left(T^{q}\left(x^{(i)}-x^{(j)}\right)\right) \leq \varepsilon / 2$ for $i, j \geq N$. In view of the K-property we can choose $i_{0} \geq N$ such that

$$
\sum_{r=1}^{\lambda_{s+1}-1}\left|x_{r}^{\left(i_{0}\right)}-x_{r}\right| p_{k}\left(e^{r}\right) \leq \frac{\varepsilon}{2} \text {. }
$$

Then for every $i \geq N$ by (2.4) we get

$$
p_{k}\left(\left(u^{(i)}\right)^{[s]}\right) \leq p_{k}\left(T^{q}\left(x^{(i)}-x^{\left(i_{0}\right)}\right)\right)+\sum_{r=1}^{\lambda_{s+1}-1}\left|x_{r}^{\left(i_{0}\right)}-x_{r}\right| p_{k}\left(e^{r}\right) \leq \varepsilon .
$$

Then by (2.5) also $p_{k}\left(u^{(i)}\right) \leq \varepsilon$ for $i \geq N$, hence $x^{(n)} \rightarrow x$ in $(X(|\lambda|, q), \tau)$, implying that $(X(|\lambda|, q), \tau)$ is complete.

Lemma 2.4. If $1 \leq p<q \leq \infty$, then $X(|\lambda|, p) \subset X(|\lambda|, q)$. 
Proof. For $p<q, n \in \mathbb{N}$ and $x \in \omega$ we get

$$
0 \leq\left[T^{q}(x)\right]_{n} \leq\left[T^{p}(x)\right]_{n} \quad(n \in \mathbb{N}) .
$$

Since $X$ is positively solid, $T^{p}(x) \in X$ implies $T^{q}(x) \in X$.

Remark 2.5. 1) Evidently $X(|\lambda|, p)=X(|\lambda|, q)$ for any $1 \leq p, q \leq \infty$ if $X^{+}=|\omega|$. We also construct an example of $X$ which satisfies the first equality, but $X^{+} \subsetneq|\omega|$. Let $\left(\lambda_{n}\right)$ be an index sequence satisfying (1.1). We set

$$
X:=\left\{z \in \omega \mid \sqrt[\lambda_{n+1}-\lambda \eta]{\left|z_{n}\right|} \rightarrow 0\right\}
$$

and note that $X$ is solid. Since

$$
\sqrt[\lambda_{n+1}-\lambda_{n}]{\left[T^{\infty}(x)\right]_{n}} \leq \sqrt[\lambda_{n+1}-\lambda_{n}]{\left[T^{1}(x)\right]_{n}} \leq \sqrt[\lambda_{n+1}-\lambda_{n}]{\left(\lambda_{n+1}-\lambda_{n}\right)\left[T^{\infty}(x)\right]_{n}}
$$

for every $n \in \mathbb{N}$, we get

$$
\lim _{n} \sqrt[\lambda_{n+1}-\lambda n]{\left[T^{\infty}(x)\right]_{n}}=\lim _{n} \sqrt[\lambda_{n+1}-\lambda n]{\left[T^{1}(x)\right]_{n}}
$$

Hence Lemma 2.4 implies $X(|\lambda|, 1)=X(|\lambda|, p)=X(|\lambda|, \infty)$ for every $p>1$.

2) For any $p, q$ with $1<p<q<\infty$ we have

$$
\ell^{\infty}(|\lambda|)=\ell^{\infty}(|\lambda|, 1) \subsetneq \ell^{\infty}(|\lambda|, p) \subsetneq \ell^{\infty}(|\lambda|, q) \subsetneq \ell^{\infty}(|\lambda|, \infty)=\ell^{\infty} .
$$

To prove $\ell^{\infty}(|\lambda|, p) \subsetneq \ell^{\infty}(|\lambda|, q)$ for $1 \leq p<q<\infty$ we define $x_{k}:=$ $1 /\left(\lambda_{n+1}-\lambda_{n}\right)^{1 / q}$ for $\lambda_{n} \leq k<\lambda_{n+1}$ and $n \in \mathbb{N}$. Then

$$
\sup _{n}\left[T^{q}(x)\right]_{n}=1, \quad \sup _{n}\left[T^{p}(x)\right]_{n}=\sup _{n}\left(\lambda_{n+1}-\lambda_{n}\right)^{(q-p) / q p}=\infty .
$$

So $x \in \ell^{\infty}(|\lambda|, q) \backslash \ell^{\infty}(|\lambda|, p)$. To verify $\ell^{\infty}(|\lambda|, q) \subsetneq \ell^{\infty}$ we consider $x=e$.

In $[4$, Proposition 2.1] it was shown that

$$
\left(\chi \cap \ell^{\infty}(|\lambda|, 1)\right)^{\alpha}=\left(\ell^{\infty}(|\lambda|, 1)\right)^{\alpha}=\ell^{1}(|\lambda|, \infty)=\left|\ell^{\infty}\right|^{\alpha}(|\lambda|, \infty) .
$$

We will prove that the same statement holds if we take $X$ instead of $\ell^{\infty}$ on assumption $\chi(X)^{\alpha}=\left(X^{+}\right)^{\alpha}$ (which is satisfied for $X=\ell^{\infty}$ ).

Lemma 2.6. Let $X$ satisfy $\chi(X)^{\alpha}=\left(X^{+}\right)^{\alpha}$. Then

1) $(\chi \cap X(|\lambda|, 1))^{\alpha}=(\chi \cap X(|\lambda|, 1))^{\beta}=\left(X^{+}\right)^{\alpha}(|\lambda|, \infty)$;

2) $(\chi \cap X(|\lambda|, \infty))^{\alpha}=(\chi \cap X(|\lambda|, \infty))^{\beta}=\left(X^{+}\right)^{\alpha}(|\lambda|, 1)$.

Proof. 1) Since $\chi \cap X(|\lambda|, 1)$ is monotone and $(\chi \cap X(|\lambda|, 1))^{\alpha} \supset$ $(X(|\lambda|, 1))^{\alpha}$, it is sufficient to prove that $(\chi \cap X(|\lambda|, 1))^{\alpha} \subset(X(|\lambda|, 1))^{\alpha}=$ $\left(X^{+}\right)^{\alpha}(|\lambda|, \infty)$. Let $y \in(\chi \cap X(|\lambda|, 1))^{\alpha} \backslash\left(X^{+}\right)^{\alpha}(|\lambda|, \infty)$. Then $u:=T^{\infty}(y) \notin$ $\left(X^{+}\right)^{\alpha}=\chi(X)^{\alpha}$, so we can find $z \in \chi \cap X$ with $\sum_{n}\left|u_{n} z_{n}\right|=\sum_{n} u_{n} z_{n}=\infty$. We put $x_{\xi_{n}}=z_{n}$ and $x_{i}:=0$ for $i \notin\left\{\xi_{k} \mid k \in \mathbb{N}\right\}$, where $\xi_{n}$ is the minimal index $i_{0} \in\left[\lambda_{n}, \lambda_{n+1}\right)$ with $\left|y_{i_{0}}\right|=\max \left\{\left|y_{i}\right| \mid \lambda_{n} \leq i<\lambda_{n+1}\right\}(n \in \mathbb{N})$. Evidently $x \in \chi \cap X(|\lambda|, 1)$. On the other hand, 


$$
\sum_{k}\left|y_{k} x_{k}\right|=\sum_{n} u_{n} z_{n}=\infty,
$$

which contradicts $y \in(\chi \cap X(|\lambda|, 1))^{\alpha}$.

2) The proof of 2) is analogous to 1) except that the definition of $x$ is now $x_{k}:=z_{n}$ for $\lambda_{n} \leq k<\lambda_{n+1}(n \in \mathbb{N})$.

REMARK 2.7. The equality $(\chi \cap X(|\lambda|, p))^{\alpha}=\left(X^{+}\right)^{\alpha}\left(|\lambda|, p^{\prime}\right)$ for $p>1$ may fail even for $X$ satisfying $\chi(X)^{\alpha}=\left(X^{+}\right)^{\alpha}$. Note that for $q \in[1, \infty)$ we get

$$
\chi \cap \ell^{\infty}(|\lambda|, q)=\left\{x \in \chi\left|\sup _{n}\right|\left\{k \in \mathbb{N} \mid x_{k}=1\right\} \cap\left[\lambda_{n}, \lambda_{n+1}\right) \mid<\infty\right\} .
$$

So by Lemma 2.6, $\left(\chi \cap \ell^{\infty}(|\lambda|, p)\right)^{\alpha}=\ell^{1}(|\lambda|, \infty)$.

We will now verify that the converse statement for Lemma 2.6 holds even if we replace 1 with $p$ and $\infty$ with $p^{\prime}$.

LemMA 2.8. If $X(|\lambda|, p)^{\alpha}=(\chi \cap X(|\lambda|, p))^{\alpha}$, then $\left(X^{+}\right)^{\alpha}=\left(\chi \cap X^{+}\right)^{\alpha}$.

Proof. It suffices to prove that $\left(\chi \cap X^{+}\right)^{\alpha} \subset\left(X^{+}\right)^{\alpha}$. So let $w \in\left(\chi \cap X^{+}\right)^{\alpha}$. Then $\sum_{k}\left|w_{k} u_{k}\right|<\infty$ for every $u \in \chi \cap X^{+}$. We set $y_{\lambda_{i}}:=w_{i}(i \in \mathbb{N})$ and $y_{k}:=0$ for $k \notin\left\{\lambda_{i} \mid i \in \mathbb{N}\right\}$. We verify that $y=\left(y_{i}\right) \in(\chi \cap X(|\lambda|, p))^{\alpha}$. Indeed, let $x \in \chi \cap X(|\lambda|, p)$ and set $u_{i}:=\tilde{x}_{\lambda_{i}}:=x_{\lambda_{i}}(i \in \mathbb{N}), \tilde{x}_{k}:=0$ for $k \notin\left\{\lambda_{i} \mid i \in \mathbb{N}\right\}$. Since $X(|\lambda|, p)$ is solid, $\tilde{x} \in \chi \cap X(|\lambda|, p)$. Then $u=\left(u_{i}\right)=T^{p}(\tilde{x}) \in \chi \cap X^{+}$. Therefore

$$
\sum_{k}\left|y_{k} x_{k}\right|=\sum_{i}\left|y_{\lambda_{i}} x_{\lambda_{i}}\right|=\sum_{i} w_{i} u_{i}<\infty .
$$

Therefore $y \in(\chi \cap X(|\lambda|, p))^{\alpha}=X(|\lambda|, p)^{\alpha}$, hence $\sum_{k}\left|y_{k} x_{k}\right|<\infty$ for every $x \in X(|\lambda|, p)$. So if we take $u \in X^{+}$and consider $x$ with $x_{\lambda_{i}}=u_{i}(i \in \mathbb{N})$ and $x_{k}=0$ for $k \notin\left\{\lambda_{i} \mid i \in \mathbb{N}\right\}$ we obtain $u=T^{p}(x)$ and

$$
\sum_{i}\left|w_{i} u_{i}\right|=\sum_{k}\left|x_{k} y_{k}\right|<\infty \text {. }
$$

Hence $w \in\left(X^{+}\right)^{\alpha}$.

THEOREM 2.9. If $\varphi \subset X$ and $\left\langle X^{+}\right\rangle$has the matrix Hahn property, then $X(|\lambda|, 1)$ and $X(|\lambda|, \infty)$ have the separable Hahn property.

Proof. First we verify that $X(|\lambda|, 1)$ has the separable Hahn property. In view of [3, Proposition 1 and Theorem 2] it is sufficient to prove that $\chi \cap X(|\lambda|, 1) \subset c_{0 A}$ implies $X(|\lambda|, 1) \subset c_{0 A}$. We define

$$
b_{n i}:=\max _{\lambda_{i} \leq k<\lambda_{i+1}}\left|a_{n k}\right| \quad(n, i \in \mathbb{N})
$$

and verify that $\chi \cap X \subset c_{0 B}$. Since $\left(a_{n k}\right)_{k} \in(\chi \cap X(|\lambda|, 1))^{\beta}$, by Lemma 2.6 
we have $\left(b_{n k}\right)_{k} \in\left(X^{+}\right)^{\alpha}(n \in \mathbb{N})$. If we suppose on the contrary that

$$
\sum_{k} b_{n_{i} k} u_{k} \geq 4 \varepsilon \quad(i \in \mathbb{N})
$$

for some $\varepsilon>0, u \in \chi \cap X$ and an index sequence $\left(n_{i}\right)$, then by the usual gliding hump argument we may choose an index sequence $\left(k_{i}\right)$ and a subsequence $\left(m_{i}\right)$ of $\left(n_{i}\right)$ such that

$$
\sum_{k=k_{p-1}+1}^{k_{p}} b_{m_{p} k} u_{k} \geq 3 \varepsilon, \quad \sum_{k=k_{p}+1}^{\infty} b_{m_{p} k} u_{k} \leq \varepsilon
$$

$$
\sum_{k=1}^{k_{p-1}} b_{m_{p} k} u_{k} \leq \varepsilon \quad(p \in \mathbb{N}) .
$$

For $p \in \mathbb{N}$ and $k \in \mathbb{N}$ with $k_{p-1}<k \leq k_{p}$ let $\xi_{k}$ denote the minimal index $j$ with $\lambda_{k} \leq j<\lambda_{k+1}$ such that $\left|a_{m_{p} j}\right|=\max \left\{\left|a_{m_{p} i}\right| \mid \lambda_{k} \leq i<\lambda_{k+1}\right\}$. We set $x_{\xi_{k}}:=\operatorname{sgn}\left(a_{m_{p} \xi_{k}}\right) u_{k}$ for $k \in \mathbb{N}$ with $k_{p-1}<k \leq k_{p}$ and $x_{i}:=0$ for $i \notin\left\{\xi_{k} \mid k \in \mathbb{N}\right\}$. Then $x \in \chi(X(|\lambda|, 1))$. Applying (2.6) for every $p \geq 2$ we have

$$
\begin{aligned}
\left|\sum_{i=1}^{\lambda_{k_{p-1}+1}-1} a_{m_{p}} x_{i}\right| & \leq \sum_{i=1}^{k_{p-1}} \max _{\lambda_{i} \leq k<\lambda_{i+1}}\left|a_{m_{p}} k\right|\left|\sum_{k=\lambda_{i}}^{\lambda_{i+1}-1} x_{k}\right|=\sum_{i=1}^{k_{p-1}} b_{m_{p} i} u_{i}<\varepsilon, \\
\left|\sum_{i=\lambda_{k_{p}+1}}^{\infty} a_{m_{p} i} x_{i}\right| & \leq \sum_{i=k_{p}}^{\infty} \max _{\lambda_{i} \leq k<\lambda_{i+1}}\left|a_{m_{p} k} k\right| \sum_{k=\lambda_{i}}^{\lambda_{i+1}-1} x_{k} \mid=\sum_{i=k_{p}}^{\infty} b_{m_{p} i} u_{i}<\varepsilon .
\end{aligned}
$$

Now for every $p \in \mathbb{N}$ we get

$$
\sum_{i} a_{m_{p} i} x_{i}=\sum_{i=1}^{\lambda_{k_{p-1}+1}-1} a_{m_{p} i} x_{i}+\sum_{k=k_{p-1}+1}^{k_{p}} b_{m_{p} k} u_{k}+\sum_{i=\lambda_{k_{p}+1}}^{\infty} a_{m_{p} i} x_{i} \geq \varepsilon,
$$

contrary to $x \in c_{0 A}$. Hence $\chi \cap X \subset c_{0 B}$, implying $X^{+} \subset c_{0 B}$.

Now for every $x \in X(|\lambda|, 1)$ we get

$$
\left|\sum_{k} a_{n k} x_{k}\right| \leq \sum_{i} \sum_{k=\lambda_{i}}^{\lambda_{i+1}-1}\left|a_{n k}\right|\left|x_{k}\right| \leq \sum_{i} b_{n i}\left[T^{1}(x)\right]_{i} \quad(n \in \mathbb{N}),
$$

so $x \in c_{0 A}$.

For $X(|\lambda|, \infty)$ we use the same idea of proof except that we define $B=$ $\left(b_{n i}\right)$ and $x$ by setting $b_{n i}:=\sum_{k=\lambda_{i}}^{\lambda_{i+1}-1}\left|a_{n k}\right|(n, i \in \mathbb{N})$ and $x_{j}:=u_{k} \operatorname{sgn}\left(a_{m_{p} j}\right)$ for $p, k, j \in \mathbb{N}$ with $k_{p-1}<k \leq k_{p}$ and $\lambda_{k} \leq j<\lambda_{k+1}$. 
REMARK 2.10. 1) Note that $X(|\lambda|, 1)$ may fail to have the Hahn property even if $\left\langle X^{+}\right\rangle$has the Hahn property. As an example consider the space $\ell^{\infty}(|\lambda|, 1)$ (cf. [4, Corollary 2.5]).

2) In view of Remark 2.7 the space $X(|\lambda|, p)$ for $p \in(1, \infty)$ fails in general to have the matrix Hahn property.

The following result demonstrates that $X(|\lambda|, 1)$ has the separable Hahn property if and only if $\left\langle X^{+}\right\rangle$does.

Proposition 2.11. If $X(|\lambda|, p)$ has the matrix Hahn property, then $\left\langle X^{+}\right\rangle$has the separable Hahn property.

Proof. Since $\left\langle X^{+}\right\rangle$is solid, by [3, Proposition 1 and Theorem 2] it is sufficient to prove that $\chi \cap\left\langle X^{+}\right\rangle \subset c_{0 B}$ implies $\left\langle X^{+}\right\rangle \subset c_{0 B}$.

Suppose on the contrary that we can find a matrix $B=\left(b_{n k}\right)$ and $u \in X^{+}$ such that $\chi \cap X^{+} \subset c_{0 B}$, but $u \notin c_{0 B}$.

We define the matrix $A=\left(a_{n k}\right)$ and the sequence $x=\left(x_{k}\right)$ by $a_{n \lambda_{i}}:=b_{n i}$, $x_{\lambda_{i}}:=u_{i}(n, i \in \mathbb{N})$ and $a_{n k}:=x_{k}:=0$ for $k \notin\left\{\lambda_{i} \mid i \in \mathbb{N}\right\}$ and $n \in \mathbb{N}$. Evidently, $x \in X(|\lambda|, p)$.

We will verify that $\chi \cap X(|\lambda|, p) \subset c_{0 A}$, but $x \notin c_{0 A}$, which would imply that $X(|\lambda|, p)$ does not have the matrix Hahn property.

To prove the first statement let $y \in \chi \cap X(|\lambda|, p)$ and set $v_{k}:=y_{\lambda_{k}}$ $(k \in \mathbb{N})$. Since $0 \leq v_{k} \leq\left[T^{p}(y)\right]_{k}(k \in \mathbb{N})$, we have $v=\left(v_{k}\right) \in \chi \cap X^{+} \subset c_{0 B}$. So

$$
\lim _{n} \sum_{i} a_{n i} y_{i}=\lim _{n} \sum_{k} a_{n \lambda_{k}} y_{\lambda_{k}}=\lim _{n} \sum_{k} b_{n k} v_{k}=0 .
$$

Hence $y \in c_{0 A}$, implying $\chi \cap X(|\lambda|, p) \subset c_{0 A}$.

On the other hand, $\left(\sum_{k} a_{n k} x_{k}\right)_{n}=\left(\sum_{k} b_{n k} u_{k}\right)_{n} \notin c_{0}$, that is, $x \notin c_{0 A}$.

\section{References}

[1] G. Bennett, J. Boos, and T. Leiger, Sequences of O's and 1's, Studia Math. 149 (2002), 75-99.

[2] J. Boos, Classical and Modern Methods in Summability, Oxford Univ. Press, New York, Oxford, 2000.

[3] J. Boos and T. Leiger, Addendum: Sequences of 0's and 1's (Studia Math. 149 (2002), 75-99), Studia Math. 171 (2005), 305-309.

[4] - - - Sequences of 0's and 1's: special sequence spaces with the separable Hahn property, Acta Math. Hungar., to appear (2006).

[5] J. Boos and M. Zeltser, Sequences of 0's and 1's. Classes of concrete 'big' Hahn spaces, Z. Anal. Anwend. 22 (2003), 819-842.

[6] K.-G. Grosse-Erdmann, The Blocking Technique, Weighted Mean Operators and Hardy's Inequality Series, Lecture Notes in Math. 1679, Springer, Berlin, 1998.

[7] C. E. Stuart and P. Abraham, Generalizations of the Nikodym boundedness and Vitali-Hahn-Saks theorems, J. Math. Anal. Appl. 300 (2004), 351-361. 
[8] M. Zeltser, J. Boos and T. Leiger, Sequences of 0's and 1's: New results via double sequence spaces, J. Math. Anal. Appl. 275 (2002), 883-899.

Matemaatika-loodusteaduskond

Tallinna Ülikool

Narva mnt. 25

EE 10120 Tallinn, Estonia

E-mail: mariaz@tlu.ee 